\title{
Xylaria spinulosa sp. nov. and $X$. atrosphaerica from southern China
}

\section{Li QR ${ }^{1,2}$, Liu LL ${ }^{2}$, Zhang $X^{2}$, Shen $X^{2}$ and Kang $\mathrm{JC}^{1^{*}}$}

${ }^{I}$ The Engineering and Research Center for Southwest Bio-Pharmaceutical Resources of National Education Ministry of China, Guizhou University Guiyang 550025, People's Republic of China

${ }^{2}$ The High Educational Key Laboratory of Guizhou Province for Natural Medicinal Pharmacology and Druggability, Guizhou Medical University, Huaxi University Town, Guian new district 550025, Guizhou, People's Republic of China

Li QR, Liu LL, Zhang X, Shen XC, Kang JC 2017 - Xylaria spinulosa sp. nov. and X. atrosphaerica from southern China. Mycosphere 8(8), 1070-1079, Doi 10.5943/mycosphere/8/8/8

\begin{abstract}
Two species of Xylaria collected from southern China are reported. Xylaria spinulosa sp. nov. is introduced as a new species based on morphology and sequence data analysis. Xylaria spinulosa differs from other species in the genus mainly by its long spines covering the surface of the stroma. Xylaria atrosphaerica is a new record for China. Descriptions and illustrations for both species are provided in this paper.
\end{abstract}

Key words - morphology - new species - phylogeny - taxonomy - Xylariales

\section{Introduction}

Xylaria belongs in the subclass Xylariomycetidae in the order Xylariales, a group which is presently undergoing considerable revision (Daranagama et al. 2015, 2016). It is not presently clear how many families will be accepted in the genus, but Xylaria Hill ex Schrank is the largest genus in the family with species having been recorded in most countries worldwide (Læssøe 1987, 1999, Ju \& Hsieh 2007, Ju et al. 2009, Liu et al. 2015, Hongsanan et al. 2017). Currently, nearly 300 estimated species are thought to belong in Xylaria (Kirk et al. 2008). Xylaria differs from other genera in Xylariaceae by producing upright, cylindrical to clavate, multi-peritheciate stromata (Maharachchikumbura et al. 2016). Most Xylaria species produce stromata on decayed dicotyledonous wood (Hsieh et al. 2010).

Species of Xylaria are difficult to identify and classify especially as stroma of a given species often varies greatly in colour, size and sometimes in general shape (Whalley 1996). Species have mainly been resolved on the basis of morphological characteristics. These characteristics are not suitable for differentiating closely related species of Xylaria, due to lack of differentiating characteristics. The genus Xylaria has great variation in morphology and may be polyphyletic. Few phylogenetic studies have been conducted to infer the relationships of the taxa of Xylaria and there is a need for a comprehensive monograph. ITS sequence data have been used to infer phylogenetic relationships among species of Xylaria by Lee et al. (2000). Hsieh et al. (2010) analyzed 114 species of Xylariaceae, based on $\alpha$-actin, $\beta$-tubulin and $\mathrm{rpb} 2$ sequence data and stated that Xylaria is a paraphyletic genus. Hsieh et al. (2010) made an important step to resolve closely related species of Xylaria.

Although there has been a high interest in Xylaria over the last 30 years (Smith et al. 2003, Tang et al. 2009, Daranagama et al. 2015, 2016, Hashemi et al. 2015, Karun \& Sridhar 2015, Srihanant et al. 2015, Kim et al. 2016), these have generally not incorporated molecular data and 
have rarely looked at type species. There have been very few reports on the genus from the Chinese mainland (Chou 1935, Tai 1979, Abe \& Liu 1995, Ma et al. 2011, Huang et al. 2014a, b). Therefore, the diversity of Xylaria in China is still underrepresented and needs further investigation (Ma et al. 2011). In the present paper, we have collected Xylaria species from China and introduce Xylaria spinulosa sp. nov. and provide a description of $X$. atrosphaerica which is a new record for China.

\section{Materials \& Methods}

\section{Isolation and morphology}

The fungal materials were collected in the forest from Hainan and Guizhou provinces in China. The methodology used for morphological examination of fungi followed that used by Stadler et al. (2004). Materials were mounted in water and Melzer's iodine reagent for examination. Asci and ascospores were examined by light microscopy (BX41, Olympus). Cultures were obtained by placing tissue of freshly collected stromata on Oatmeal agar (Ju et al. 2009). Total DNA extraction and sequencing of ITS rDNA, $\alpha$-actin gene (act) and RNA polymerase II second largest subunit genes (rpb2) follow Ju \& Hsieh (2007). For PCR amplification of $\beta$-tubulin gene (tub2), primers T1/T2, Bt2a/Bt2b were used (Ju \& Hsieh 2007). The herbarium and living culture are deposited in the Collection of Guizhou University (GZUCC) and Kunming Institute of Botany (KUN), Chinese Academy of Sciences.

\section{Phylogenetic analyses}

All sequences used in this paper were gained from recent publications and are listed in Table 1. The alignments are available in TreeBASE (www.treebase.org/treebase-web/home.html) under ID15112 for ITS alignment and ID 20686 for act-rpb2-tub2 alignment. A-actin, $\beta$-tubulin and $\mathrm{rpb} 2$ sequence data were assembled using the alignment program BioEdit (Hall 1999) and ClustalX (Thompson et al. 1997). Alignment was manually adjusted to achieve the maximum alignment and to minimize gaps. Maximum parsimony was applied to the act-tub2-rpb2 dataset. All characters were assessed as independent, unordered, and equally weighted. Gaps were treated as missing characters. Biscogniauxia arima was used as the outgroup taxon (Hsieh et al. 2010). Maximum parsimony analysis was performed using PAUP (Phylogenetic Analysis Using Parsimony) v.4.0b10 (Felsenstein 1985, Swofford 2002).

\section{Results}

Using ITS rDNA sequences, our initial BLAST searches suggested that our sequences were most closely related to those of the taxa in Xylaria polymorpha complex (Hsieh et al. 2010). Act, $\mathrm{Rpb} 2$ and tub2 genes sequences were used to identify those species.

Phylogenetic analyses

A phylogenetic study using three combined loci (Rpb2, act and tub2) was conducted. All characters and gaps have equal weight. A total of 47 taxa were included in the analysis. Total length of the alignment had 1892 characters, in which 144 of those characters were variable, 935 characters were constant, 813 parsimony-informative characters.

The result of the phylogenetic analysis is shown in Fig. 1. The appearance of Xylaria spinulosa $(100 \% \mathrm{BS})$ was reasonably well-supported as sister clade of X. haemorrhoidalis Berk. \& Broome. The closest relatives to $X$. spinulosa, clustered basal to this subclade, were $X$. scruposa (Fr.: Fr.) Fr., X. atrosphaerica (Cooke \& Massee) Callan \& J. D. Rogers, X. polymorpha (Pers.: Fr.) Grev., X. cf. glebulosa (Ces.) Y.-M. Ju \& J. D. Rogers, X. ophiopoda Sacc. X. schweinitzii Berk. \& M. A. Curtis, X. globosa (Spreng. ex Fr.: Fr.) Mont. and the earliest diverging lineage is represented by Xylaria enterogena (Mont.) Fr. and X. telfairii (Berk.) Fr. supported by the bootstrap analysis (100\% BS). Xylaria atrosphaerica GZUCC14028 and X. atrosphaerica 91111214 HAST clustered at a short branch with high bootstrap support (100\% 
Table 1 Strains used in phylogenetic analyses and their corresponding GenBank accession numbers.

\begin{tabular}{|c|c|c|c|c|c|}
\hline \multirow[t]{2}{*}{ Taxon } & \multirow{2}{*}{ Origin and strains } & \multicolumn{3}{|c|}{ GenBank accession number } & \multirow{2}{*}{ References } \\
\hline & & Rpb2 & $\alpha$-actin & $\beta$-tubulin & \\
\hline $\begin{array}{l}\text { Amphirosellinia } \\
\text { fushanensis }\end{array}$ & $\begin{array}{l}\text { Taiwan, } 911111209 \\
\text { HAST }\end{array}$ & GQ848339 & GQ452360 & GQ495950 & Ju et al. 2004 \\
\hline A. nigrospora & $\begin{array}{l}\text { Taiwan, } 91092308 \\
\text { HAST }\end{array}$ & GQ848340 & GQ452361 & GQ495951 & Ju et al. 2004 \\
\hline Astrocystis bambusae & $\begin{array}{l}\text { Taiwan, } 89021904 \\
\text { HAST }\end{array}$ & GQ844836 & GQ449239 & GQ495942 & $\begin{array}{l}\text { Hsieh et al. } \\
2010\end{array}$ \\
\hline As. mirabilis & $\begin{array}{l}\text { Taiwan, } 94070803 \\
\text { HAST }\end{array}$ & GQ844835 & GQ449238 & GQ495941 & $\begin{array}{l}\text { Hsieh et al. } \\
2010\end{array}$ \\
\hline As. sublimbata & $\begin{array}{l}\text { Taiwan, } 89032207 \\
\text { HAST }\end{array}$ & GQ844834 & GQ449236 & GQ495940 & $\begin{array}{l}\text { Hsieh et al. } \\
2010\end{array}$ \\
\hline Biscogniauxia arima & Mexico, YMJ 122 & GQ304736 & AY951784 & AY951672 & $\begin{array}{l}\text { Ju et al. 1998; } \\
\text { Hsieh et al. } \\
2005\end{array}$ \\
\hline $\begin{array}{l}\text { Discoxylaria } \\
\text { myrmecophila }\end{array}$ & Mexico, 169 HAST & GQ844819 & GQ438747 & GQ487710 & $\begin{array}{l}\text { Rogers et al. } \\
1995\end{array}$ \\
\hline $\begin{array}{l}\text { Stilbohypoxylon } \\
\text { elaeicola }\end{array}$ & $\begin{array}{l}\text { Taiwan, } 94082615 \\
\text { HAST }\end{array}$ & GQ844827 & GQ438754 & GQ495933 & $\begin{array}{l}\text { Hsieh et al. } \\
2010\end{array}$ \\
\hline S. quisquiliarum & $\begin{array}{l}\text { French Guiana, } \\
91111209 \text { HAST }\end{array}$ & GQ853020 & EF025590 & EF025605 & $\begin{array}{l}\text { Rogers \& Ju, } \\
1997 \text {; Ju et al. } \\
2007\end{array}$ \\
\hline S. quisquiliarum & Taiwan, YMJ 172 & GQ853021 & EF025591 & EF025606 & Ju et al. 2007 \\
\hline Xylaria allantoidea & $\begin{array}{l}\text { Taiwan, } 94042903 \\
\text { HAST }\end{array}$ & GQ848356 & GQ452377 & GQ502692 & $\begin{array}{l}\text { Hsieh et al. } \\
2010\end{array}$ \\
\hline X. apoda & $\begin{array}{l}\text { Taiwan, } 90080804 \\
\text { HAST }\end{array}$ & GQ844823 & GQ438751 & GQ495930 & $\begin{array}{l}\text { Hsieh et al. } \\
2010\end{array}$ \\
\hline$X$. atrosphaerica & $\begin{array}{l}\text { Taiwan, } 91111214 \\
\text { HAST }\end{array}$ & GQ848342 & GQ452363 & GQ495953 & $\begin{array}{l}\text { Hsieh et al. } \\
2010\end{array}$ \\
\hline X. badia & $\begin{array}{l}\text { Taiwan, } 95070101 \\
\text { HAST }\end{array}$ & GQ844833 & GQ449235 & GQ495939 & $\begin{array}{l}\text { Hsieh et al. } \\
2010\end{array}$ \\
\hline$X$. berteri & Taiwan, YMJ 90112623 & GQ848362 & AY951874 & AY951763 & $\begin{array}{l}\text { Hsieh et al. } \\
2005\end{array}$ \\
\hline X. castorea & $\begin{array}{l}\text { New Zealand, PDD } \\
47417\end{array}$ & GQ853018 & GQ455447 & GQ502703 & $\begin{array}{l}\text { Hsieh et al. } \\
2010\end{array}$ \\
\hline$X$. cf. castorea & $\begin{array}{l}\text { Taiwan, } 91092303 \\
\text { HAST }\end{array}$ & GQ853019 & GQ455448 & GQ502704 & $\begin{array}{l}\text { Hsieh et al. } \\
2010\end{array}$ \\
\hline X. cf. glebulosa & $\begin{array}{l}\text { French West Indies, } 431 \\
\text { HAST }\end{array}$ & GQ848345 & GQ452366 & GQ495956 & $\begin{array}{l}\text { Hsieh et al. } \\
2010\end{array}$ \\
\hline X. cf. heliscus & $\begin{array}{l}\text { Taiwan, } 88113010 \\
\text { HAST }\end{array}$ & GQ848355 & GQ452376 & GQ502691 & $\begin{array}{l}\text { Hsieh et al. } \\
2010\end{array}$ \\
\hline X. crozonensis & France, 398 HAST & GQ848361 & GQ455441 & GQ502697 & $\begin{array}{l}\text { Hsieh et al. } \\
2010\end{array}$ \\
\hline X. cubensis & $\begin{array}{l}\text { Papua New Guinea, } 860 \\
\text { JDR }\end{array}$ & GQ853017 & GQ455446 & GQ502702 & Gucht, 1995 \\
\hline X. culleniae & Thailand, 189 JDR & GQ844829 & GQ438756 & GQ495935 & $\begin{array}{l}\text { Hsieh et al. } \\
2010\end{array}$ \\
\hline X. curta & $\begin{array}{l}\text { French West Indies, } 494 \\
\text { HAST }\end{array}$ & GQ844831 & GQ449233 & GQ495937 & $\begin{array}{l}\text { Hsieh et al. } \\
2010\end{array}$ \\
\hline$X$. digitata & Ukraine, 919 HAST & GQ848338 & GQ449245 & GQ495949 & $\begin{array}{l}\text { Hsieh et al. } \\
2010\end{array}$ \\
\hline X. enterogena & $\begin{array}{l}\text { French Guiana, } 785 \\
\text { HAST }\end{array}$ & GQ848349 & GQ452370 & GQ502685 & $\begin{array}{l}\text { Hsieh et al. } \\
2010\end{array}$ \\
\hline X.feejeensis & $\begin{array}{l}\text { Taiwan, } 92092013 \\
\text { HAST }\end{array}$ & GQ848336 & GQ449243 & GQ495947 & $\begin{array}{l}\text { Hsieh et al. } \\
2010\end{array}$ \\
\hline X. feejeensis & Thailand, 180 JDR & GQ848335 & GQ449242 & GQ495946 & $\begin{array}{l}\text { Hsieh et al. } \\
2010\end{array}$ \\
\hline X. frustulosa & $\begin{array}{l}\text { Taiwan, } 92092010 \\
\text { HAST }\end{array}$ & GQ844838 & GQ449240 & GQ495944 & $\begin{array}{l}\text { Hsieh et al. } \\
2010\end{array}$ \\
\hline
\end{tabular}


Table 1 Strains used in phylogenetic analyses and their corresponding GenBank accession numbers.

\begin{tabular}{|c|c|c|c|c|c|}
\hline \multirow[t]{2}{*}{ Taxon } & \multirow{2}{*}{ Origin and strains } & \multicolumn{3}{|c|}{ GenBank accession number } & \multirow{2}{*}{ References } \\
\hline & & Rpb2 & $\alpha$-actin & $\beta$-tubulin & \\
\hline X. globosa & $\begin{array}{l}\text { French West Indies, } 775 \\
\text { HAST }\end{array}$ & GQ848348 & GQ452369 & GQ502684 & $\begin{array}{l}\text { Hsieh et al. } \\
2010\end{array}$ \\
\hline X. haemorrhoidalis & $\begin{array}{l}\text { Taiwan, } 89041207 \\
\text { HAST }\end{array}$ & GQ848347 & GQ452368 & GQ502683 & $\begin{array}{l}\text { Hsieh et al. } \\
2010\end{array}$ \\
\hline X. ianthinovelutina & $\begin{array}{l}\text { French West Indies, } 553 \\
\text { HAST }\end{array}$ & GQ844828 & GQ438755 & GQ495934 & $\begin{array}{l}\text { Hsieh et al. } \\
2010\end{array}$ \\
\hline X. intracolorata & $\begin{array}{l}\text { Taiwan, } 90080402 \\
\text { HAST }\end{array}$ & GQ502690 & GQ452375 & GQ502690 & $\begin{array}{l}\text { Hsieh et al. } \\
2010\end{array}$ \\
\hline X. juruensis & $\begin{array}{l}\text { Taiwan, } 92042501 \\
\text { HAST }\end{array}$ & GQ844825 & GQ438753 & GQ495932 & $\begin{array}{l}\text { Hsieh et al. } \\
2010\end{array}$ \\
\hline X. laevis & $\begin{array}{l}\text { French West Indies, } 419 \\
\text { HAST }\end{array}$ & GQ848359 & GQ455439 & GQ502695 & $\begin{array}{l}\text { Hsieh et al. } \\
2010\end{array}$ \\
\hline $\begin{array}{l}\text { X. luteostromata } \text { var. } \\
\text { macrospora }\end{array}$ & $\begin{array}{l}\text { French West Indies, } 508 \\
\text { HAST }\end{array}$ & GQ848352 & GQ452373 & GQ502688 & $\begin{array}{l}\text { Hsieh et al. } \\
2010\end{array}$ \\
\hline X. ophiopoda & $\begin{array}{l}\text { Taiwan, } 93082805 \\
\text { HAST }\end{array}$ & GQ848344 & GQ452365 & GQ495955 & $\begin{array}{l}\text { Hsieh et al. } \\
2010\end{array}$ \\
\hline X. oxyacanthae & USA, 93082805 HAST & GQ844820 & GQ438748 & GQ495927 & $\begin{array}{l}\text { Hsieh et al. } \\
2010\end{array}$ \\
\hline X. palmicola & New Zealand, 604 PDD & GQ844822 & GQ438750 & GQ495929 & $\begin{array}{l}\text { Hsieh et al. } \\
2010\end{array}$ \\
\hline$X$. phyllocharis & $\begin{array}{l}\text { French West Indies, } 528 \\
\text { HAST }\end{array}$ & GQ844832 & GQ449234 & GQ495938 & $\begin{array}{l}\text { Hsieh et al. } \\
2010\end{array}$ \\
\hline X. plebeja & $\begin{array}{l}\text { Taiwan, } 91122401 \\
\text { HAST }\end{array}$ & GQ848353 & GQ452374 & GQ502689 & $\begin{array}{l}\text { Hsieh et al. } \\
2010\end{array}$ \\
\hline X. polymorpha & USA, 1012 JDR & GQ848343 & GQ452364 & GQ495954 & $\begin{array}{l}\text { Hsieh et al. } \\
2010\end{array}$ \\
\hline$X$. regalis & $\begin{array}{l}\text { Taiwan, } 92072001 \\
\text { HAST }\end{array}$ & GQ848357 & GQ452378 & GQ502693 & $\begin{array}{l}\text { Hsieh et al. } \\
2010\end{array}$ \\
\hline X. schweinitzii & $\begin{array}{l}\text { Taiwan, } 92092023 \\
\text { HAST }\end{array}$ & GQ848346 & GQ452367 & GQ495957 & $\begin{array}{l}\text { Hsieh et al. } \\
2010\end{array}$ \\
\hline X. scruposa & $\begin{array}{l}\text { French West Indies, } \\
\text { 497HAST }\end{array}$ & GQ848341 & GQ452362 & GQ495952 & $\begin{array}{l}\text { Hsieh et al. } \\
2010\end{array}$ \\
\hline$X$. telfairii & $\begin{array}{l}\text { French West Indies, } 421 \\
\text { HAST }\end{array}$ & GQ848350 & GQ452371 & GQ502686 & $\begin{array}{l}\text { Hsieh et al. } \\
2010\end{array}$ \\
\hline X. spinulosa & $\begin{array}{l}\text { Hainan, China, } \\
\text { GZUCC13016 }\end{array}$ & KM236098 & KM236097 & KM236099 & This study \\
\hline X. atrosphaerica & $\begin{array}{l}\text { Guizhou, China, } \\
\text { GZUCC14028 }\end{array}$ & KY677915 & KY677916 & KY677917 & This study \\
\hline
\end{tabular}

JDR: Herbarium of Jack D. Rogers; HAST: Herbarium, Research Center for Biodiversity, Academia Sinica, Taipei; YMJ: Herbarium of Yu-Min Ju; PDD: Landcare Research

\section{Taxonomy}

Xylaria spinulosa Q.R. Li \& J.C. Kang, sp. nov.

MycoBank - MB513099; Facesoffungi number - FoF 03180

(Figs 2, 3)

Etymology - in reference to the long thorns covering the stromatal surface.

Holotype - GZUH13016

Saprobic on dead tree trunk, forming on the host surface. Sexual morph: Stromata upright, usually solitary, unbranched, cylindrical or clavate, fertile at apex, texture soft, internally white, whole stromata up to $1.8-2.5 \mathrm{~cm}$ long $\times 0.3-0.5 \mathrm{~cm}$ broad, $0.5-1 \mathrm{~cm}$ long at stipes. Surface of stromata black, covered with long soft hairs. Stalk well defined and easy to distinguish. Perithecia 0.3-0.5 mm diam., sphaerical, embedded, closely arranged. Ostioles inconspicuous, not clear. Asci 80-214 × 8-12 $\mathrm{m} \quad(157 \times 10.2 \mu \mathrm{m}, \mathrm{n}=20)$, unitunicate, 8-spored, cylindrical, long-stipitate, the spore-bearing part $60-170 \mu \mathrm{m}$ long. Apical ring $8.5-11.5 \mu \mathrm{m}$ high $\times 5-6 \mu \mathrm{m}$ broad, blue in Melzer's reagent, urn-shaped, 8.5-11.5 $\mu \mathrm{m}$ high $\times 5-6 \mu \mathrm{m}$ broad. Ascospores $19-23 \times 6.5-8.5 \mu \mathrm{m}$ $(21.3 \times 7.6 \mu \mathrm{m}, \mathrm{n}=30)$, dark brown to black, unicellular, ellipsoid to inequilateral, with broadly 
rounded ends, smooth-walled. Germ slit half spore-length, oblique to sigmoid, lacking sheath and appendage. Asexual morph: Undetermined.

Culture characteristics - Colonies on OA (Fig. 3) cover a 9-cm plate in 3-4 weeks. Mycelia white at first, velvety, soon becoming grey and finally become to black and developing a secondary, aerial mycelium, reverse of cultures a little black.

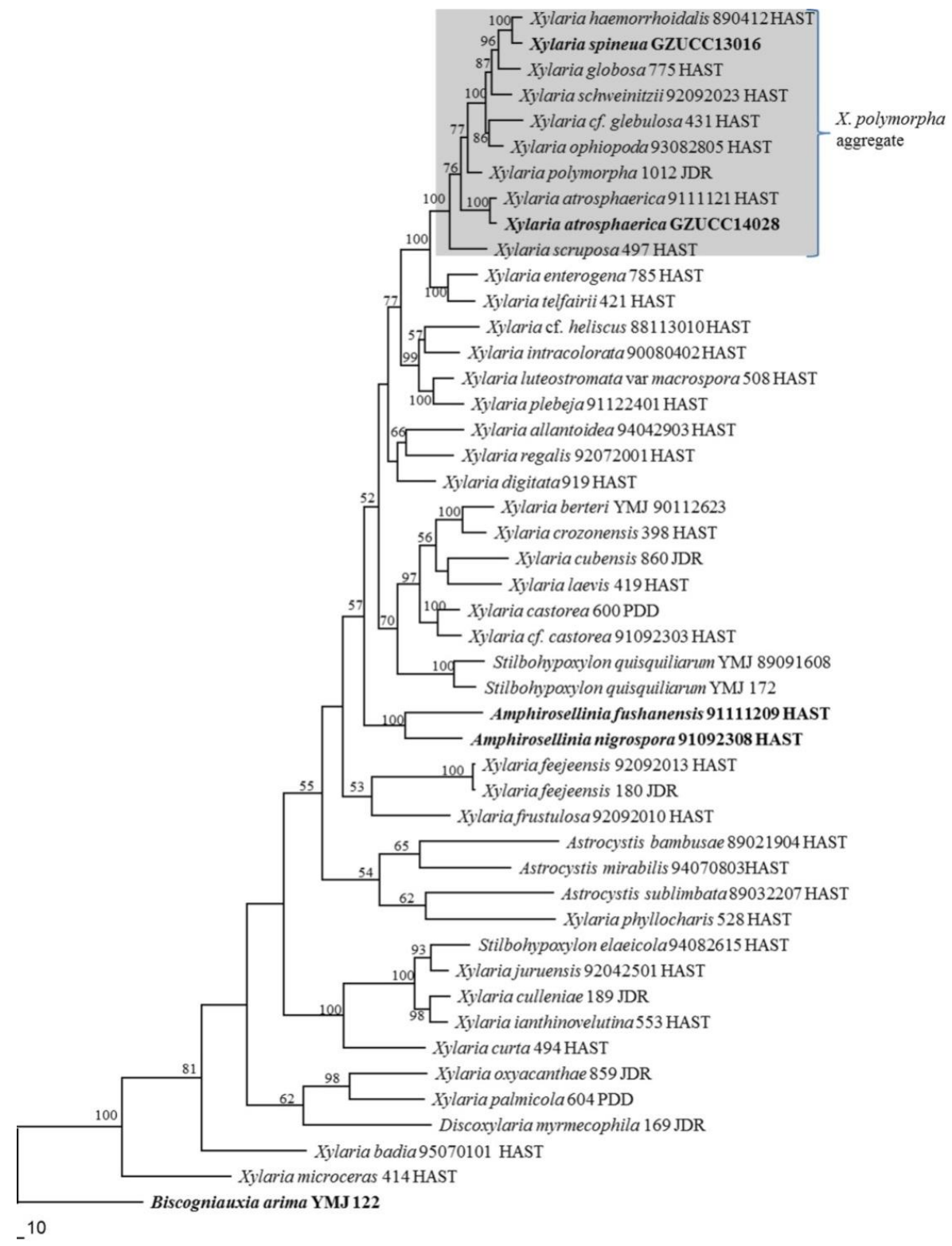

Fig. 1 - MP tree inferred from the analysis of act, $\mathrm{rpb} 2$ and tub2 genes sequences. $\mathrm{CI}=0.319$, $\mathrm{RI}=0.481, \mathrm{RC}=0.154, \mathrm{HI}=0.681$. Bootstrap support values based on 1000 replicates are indicated (above 50\%) at the branches at nodes. Sequences labelled in bold were type and our cultures. The tree was rooted to Biscogniauxia arima YMJ 122.

No pigments were observed. Stromata arising after 3 weeks of incubation, cylindrical, unbranched, up to $3 \mathrm{~cm}$ high and $2 \mathrm{~mm}$ diam.

Material examined - CHINA, Hainan Province, Wuzhishan City, Wuzhishan Nature Reserve, on deadwood of unknown plant, March 2013, Q.R. Li, WZS3 (GZUH 13016, holotype; HKAS 99544, isotype); ex-type living culture, GZUCC 13016. 
Xylaria atrosphaerica (Cooke \& Massee) Callan \& J.D. Rogers, Mycotaxon 36 (2): 349 (1990).

(Fig. 4) MycoBank MB 127393

Saprobic on dead tree trunk, forming on the host surface. Sexual morph: Stromata semiglobular or irregularly globose, pulvinate, $0.5-1.5 \mathrm{~mm}$ high $\times 1.5-3 \mathrm{~mm}$ diam., with flattened or slightly convex top, grey dark to black, internally white. Surface cracked into small plates and perithecial mounds inconspicuous.

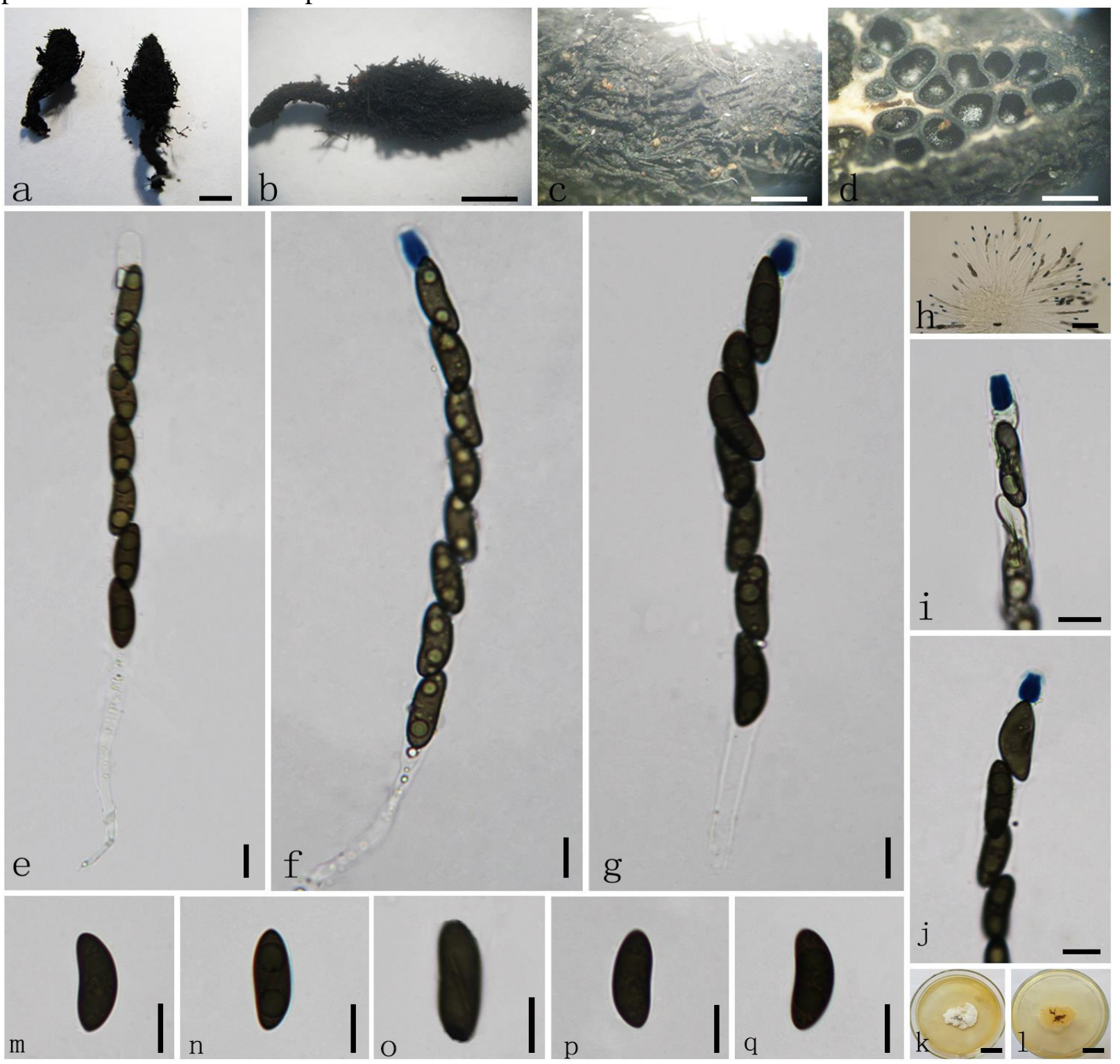

Fig. 2 - Xylaria spinulosa (GZUH13016, holotype). a-c. Stromata. d. Perithecia. e-h. Asci. I, J. Ascal apical apparatus (stained in Melzer's reagent). $\mathbf{m}-\mathbf{q}$. Ascospore. k, l. Culture on PDA. Scale bars: $\mathrm{a}, \mathrm{b}=3 \mathrm{~mm}, \mathrm{c}=1 \mathrm{~mm}, \mathrm{~d}=500 \mu \mathrm{m}, \mathrm{e}-\mathrm{g}=10 \mu \mathrm{m}, \mathrm{h}=50 \mu \mathrm{m}, \mathrm{i}-\mathrm{q}=10 \mu \mathrm{m}, \mathrm{k}, \mathrm{l}=20$ $\mathrm{mm}$.

Perithecia $0.2-0.5 \mathrm{~mm}$ diam., completely immersed, sphaerical. Ostioles inconspicuous. Asci $142-215 \times 12.5-18.5 \mu \mathrm{m}(188 \times 15.5 \mu \mathrm{m}, \mathrm{n}=30)$, unitunicate, 8-spored, cylindrical, longstipitate, the spore-bearing part $120-170 \mu \mathrm{m}$ long. Apical ring bluing in Melzer's iodine reagent, urn-shaped, 7.5-9 $\mu \mathrm{m}$ high $\times 5-6.5 \mu \mathrm{m}$ broad. Ascospores 18.5-24 $\times 6.5-9.5 \mu \mathrm{m}(22.5 \times 8 \mu \mathrm{m}$, $\mathrm{n}=30$ ), dark brown to black, unicellular, ellipsoid-inequilateral or crescent to navicular, with ends slightly pinched, smooth, with oblique to spiral germ slit, nearly $1 / 2$ spore-length, lacking sheath and appendage. Asexual morph: Undetermined.

Habitat/Distribution - Known from Guiana, Indonesia and China.

Material examined - CHINA. Guizhou Province, Tongren City, Fanjingshan Nature Reserve, on deadwood of unknown plant, July 2014, Q.R. Li, FJS 19 (GZUH14028, HKAS 99545); 


\section{Discussion}

Phylogenetic analysis (Fig. 1) showed that X. spinulosa clustered with species in the $X$. polymorpha complex (Hsieh et al. 2010).
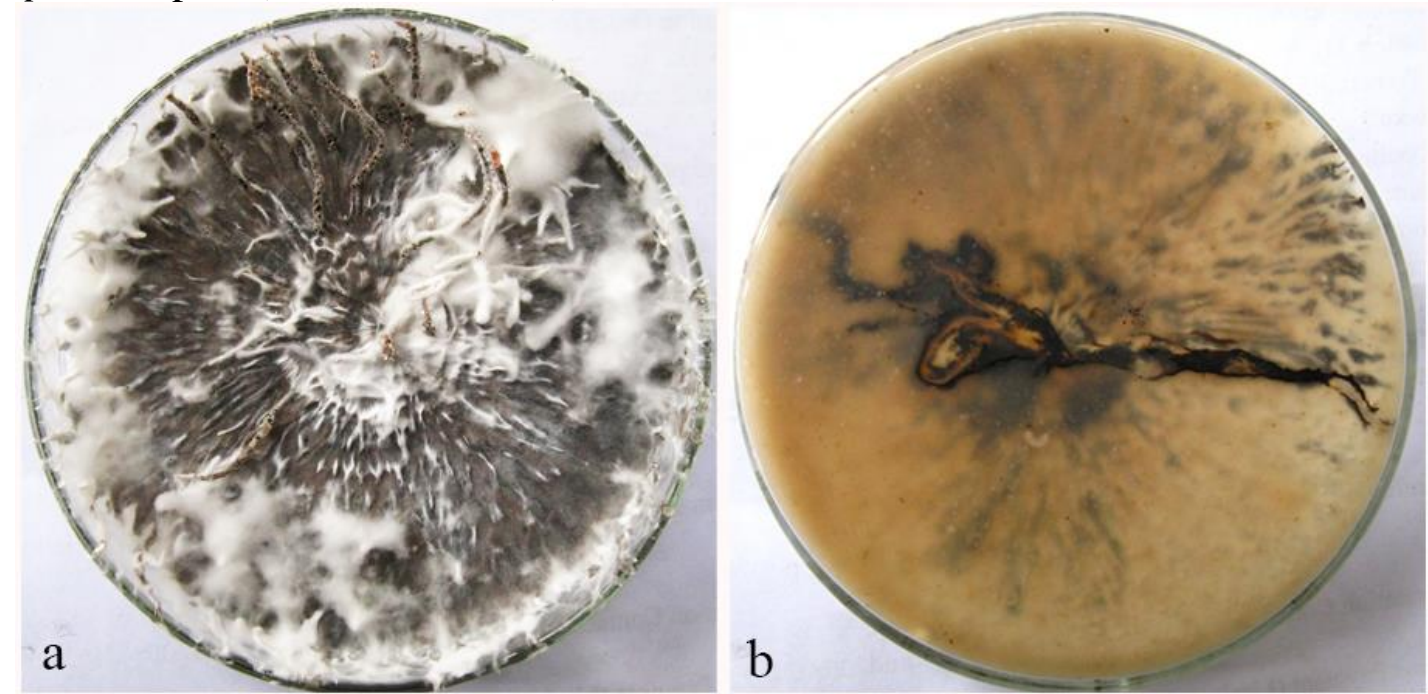

Fig. 3 - Culture of Xylaria spinulosa on OA after 4 weeks. a. Culture from above. b. Culture from below.

Taxa within the $X$. polymorpha complex formed a monophyletic group with high bootstrap support. The species were discriminated from the most common known representatives of Xylaria. Xylaria spinulosa is most closely related to X. haemorrhoidalis Berk. \& Broome according to the best-scoring maximum parsimony tree (Fig. 1). Morphologically, X. haemorrhoidalis Berk. \& Broome has subglobose stromata, and stromatal surface cracked into fine scales without long thorns. Xylaria spinulosa has upright, stipitate, woody to leathery stromata. Ascomata of $X$. spinulosa are embedded immediately beneath the surface. Species of $X$. polymorpha complex have a stromatal surface cracked into fine scales and 20-30 $\mu \mathrm{m}$ long ascospores (Ju et al. 2009). Xylaria spinulosa, however, differs from other species of the X. polymorpha complex in having long thorns on the surface of clavate stromata (Hsieh et al. 2010). Ascospores with half spore-length long germ slit from $X$. spinulosa also separates from other species in the $X$. polymorpha complex which are characterized by bearing a less than spore length germ slit (Rogers and Callan 1986).

The asexual morph of Penzigia atrosphaerica collected from Indonesia was described by Rogers et al. (1987). Based on primarily on the nature of cultures, Callan and Rogers (1990) asserted that most species should be accommodated in Xylaria and Hypoxylon and placed this species in the genus Xylaria. GZUCC14028 indicated a rather close relationship to the other sequences included X. polymorpha complex. Phylogenetic analysis (Fig. 1) showed GZUCC14028 is much closer to Xylaria atrosphaerica 91111214 HAST. Morphologically, our species has same characters with $X$. atrosphaerica and is a new record from China. Xylaria atrosphaerica was described from Guiana, Indonesia.

\section{Acknowledgements}

The authors would like thank Haixia Ma in Chinese academy of tropical agricultural sciences for her comments. This work was funded by the grants of National Natural Science Foundation of China (Grants Nos. 31460011\& 30870009), the agricultural science and technology foundation of Guizhou province, China (Grant No. NY [2013] 3042), the fund of high level innovation talents [No. 2015-4029], the fund of innovation team of Guizhou province [No. 20154025], the fund of innovated team of the education department of Guizhou province [No. 2014-31] and the program for new century excellent talents in university [No. NCET-13-0747]. 

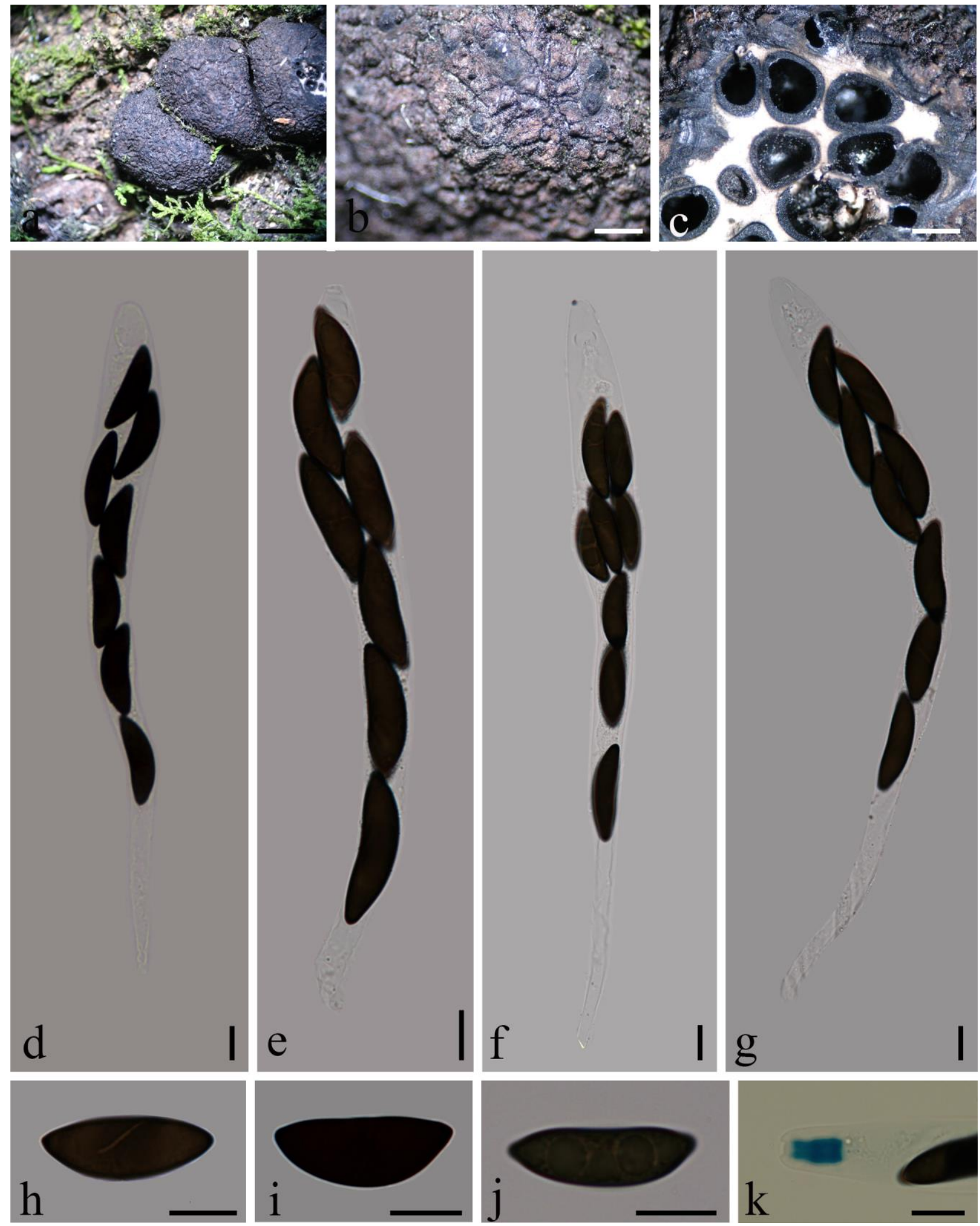

Fig. 4 - Xylaria atrosphaerica (GZUH14028). a, b. Stromata on the host. c. Vertical section of stroma. d-g. Asci with ascospores. $\mathrm{h}-\mathrm{j}$. Ascospores. k. Ascal apical apparatus and ascospores. Scale bars: $\mathrm{a}=3 \mathrm{~mm}, \mathrm{~b}, \mathrm{c}=200 \mu \mathrm{m}, \mathrm{d}-\mathrm{k}=10 \mu \mathrm{m}$.

\section{References}

Abe Y, Liu Z. 1995 - An annotated list of xylariaceous and diatrypaceous fungi collected from Mt. Fengyangshan and Mt. Baishanzu, Zhejiang Prov. in East China. Bulletin of the National 
Science Museum, Tokyo, Series B 21, 75-86.

Callan BE, Rogers JD. 1990 - Teleomorph-anamorph connections and correlations in some Xylaria species. Mycotaxon 36, 343-369.

Chou ZH. 1935 - Notes on some fungi from Kweichow. Bulletin of the Fan Memorial Institute of Biology (Botany) 6, 161-166.

Daranagama DA, Camporesi E, Liu XZ, Bhat DJ et al. 2016 - Tristratiperidium microsporum, gen. et sp. nov. (Xylariales) on dead leaves of Arundo plinii. Mycological Progress 15, 8.

Daranagama DA, Camporesi E, Tian Q, Liu XZ et al. 2015 - Anthostomella is polyphyletic comprising several genera in Xylariaceae. Fungal Diversity 73, 203-238.

Felsenstein J. 1985 - Confidence intervals on phylogenetics: an approach using bootstrap. Evolution 39, 783-791.

Gucht KVD. 1995 - Illustrations and descriptions of xylariaceous fungi collected in Papua New Guinea. Bulletin du Jardin Botanique National de Belgique 64, 219-403.

Hall TA. 1999 - Bioedit: a user-friendly biological sequence alignment editor and analysis program for Windows 95/98/NT. Nucleic Acids Symposium Series 41, 95-98.

Hashemi SA, Zare R, Khodaparast SA, Elahinia SA. 2015 - A new Xylaria species from Iran. Mycologia Iranica 2, 1-10.

Hsieh HM, Ju YM, Rogers JD. 2005 - Molecular phylogeny of Hypoxylon and closely related genera. Mycologia 97, 914-923.

Hsieh HM, Lin CR, Fang MJ, Rogers JD et al. 2010 - Phylogenetic status of Xylaria subgenus Pseudoxylaria among taxa of the subfamily Xylarioideae (Xylariaceae) and phylogeny of the taxa involved in the subfamily. Molecular Phylogenetics and Evolution 54, 957-969.

Huang G, Guo L, Liu N. 2014a - Two new species of Xylaria and X. diminuta new to China. Mycotaxon 129, 149-152.

Huang G, Guo L, Liu N. 2014b - Xylaria byttneriae sp. nov. from Yunnan Province in China. Mycosystema 33, 567-570.

Ju YM, Hsieh HM. 2007 - Xylaria species associated with nests of Odontotermes formosanus in Taiwan. Mycologia 99, 936-957.

Ju YM, Hsieh HM, Ho MC, Szu DH et al. 2007 - Theissenia rogersii sp. nov. and phylogenetic position of Theissenia. Mycologia 99, 612-621.

Ju YM, Hsieh HM, Vasilyeva L, Akulov A. 2009 - Three new Xylaria species from Russian Far East. Mycologia 101, 548-553.

Ju YM, Rogers JD, Hsieh HM. 2004 - Amphirosellinia gen. nov. and a new species of Entoleuca. Mycologia 96, 1393-1402.

Ju YM, Rogers JD, San Martín F, Granmo A. 1998 - The genus Biscogniauxia. Mycotaxon 66, 198.

Karun NC, Sridhar KR. 2015 - Xylaria complex in the South-Western India. Plant Pathology \& Quarantine 5, 83-96.

Kim CS, Jo JW, Kwag YN, Oh SO et al. 2016 - New Records of Xylaria Species in Korea: X. ripicola sp. nov. and $X$. tentaculata. Mycobiology 44, 21-28.

Kirk PF, Cannon PF, Minter DW, Stalpers JA, (eds). 2008 - Dictionary of the fungi, 10th edn. CABI, Egham CABI Bioscience, CBS, Landcare Research.

Læssøe T. 1987 - Xylaria corniformis reconsidered. Mycotaxon 30, 81-85.

Læssøe T. 1999 - The Xylaria comosa complex. Kew Bulletin 54, 605-619.

Lee JS, Ko KS, Jung HS. 2000 - Phylogenetic analysis of Xylaria based on nuclear ribosomal ITS1-5.8S-ITS2 sequences. FEMS Microbiology Letters 187, 89-93.

Liu JK, Hyde KD, Jones EBG, Ariyawansa HA et al. 2015 - Fungal diversity notes 1-110: taxonomic and phylogenetic contributions to fungal species. Fungal Diversity 72, 1-197.

Maharachchikumbura SSN, Hyde KD, Jones EBG, McKenzie EHC et al. 2016 - Families of Sordariomycetes. Fungal Diversity 79, 1-317.

Ma HX, Vasilyeva L, Li Y. 2011 - A new species of Xylaria from China. Mycotaxon 116, 151-155. Rogers JD, Callan BE. 1986 - Xylaria polymorpha and its allies in continental United States. 
Mycologia 78, 391-400.

Rogers JD, Callan BE, Samuels GJ. 1987 - The Xylariaceae of the rain forests of north Sulawesi (Indonesia). Mycotaxon 29, 113-172.

Rogers JD, Ju YM. 1997 - The genus Stilbohypoxylon. Mycological Research 101: 135-138.

Rogers JD, Ju YM, San MGF. 1995 - Discoxylaria myrmecophila and its Hypocreodendron anamorph. Mycologia 87, 41-45.

Smith GJD, Liew ECY, Hyde KD. 2003 - The Xylariales: A monophyletic order containing 7 families. Fungal Diversity 13,175-208.

Srihanant N, Petcharat V, Vasilyeva LN. 2015 - Xylaria thailandica - a new species from southern Thailand. Mycotax on 130, 227-231.

Stadler M, Wollweber H, Jäger W, Briegert M et al. 2004 - Cryptic species related to Daldinia concentrica and D. eschscholzii, with notes on D. bakeri. Mycological Research 108, 257273.

Swofford DL. 2002 - PAUP: Phylogenetic analysis using parsimony. Version 4.0 b10, Sinauer Associates, Sunderland.

Tai FL. 1979 - Sylloge fungorum Sinicorum. Science Press, Peking. 1527.

Tang A, Jeewon R, Hyde KD. 2009 - A re-evaluation of the evolutionary relationships within the Xylariaceae based on ribosomal and protein-coding gene sequences. Fungal Diversity 34, $127-155$.

Thompson JD, Gibson TJ, Plewniak F, Jeanmougin F et al. 1997 - The ClustalX Windows interface: flexible strategies for multiple sequence alignment aided by quality analysis tools. Nucleic Acids Research 24, 4876-4882.

Whalley AJS. 1996 - The xylariaceous way of life. Mycological Research 100, 897-922. 